\title{
An Enhanced Modal Approach for Large Deformation Modeling of Wing-Like Structures
}

\author{
Markus Ritter* \\ DLR - Institute of Aeroelasticity, Bunsenstraße 10, 37073 Göttingen, Germany \\ Carlos E.S. Cesnik ${ }^{\dagger}$ \\ University of Michigan, Ann Arbor, Michigan 48109-2140 \\ Wolf Reiner Krüger $\ddagger$ \\ DLR - Institute of Aeroelasticity, Bunsenstraße 10, 37073 Göttingen, Germany
}

\begin{abstract}
A new modal-based method that captures the geometric nonlinear effects that arise in the regime of large deformations of wing-like structures is presented. The most limiting factors of the modal approach are the linear force-displacement relationship and the representation of the nodal displacement field based on normal modes. The proposed extension includes stiffness terms that cubically depend on the generalized coordinates. The structural deformation is calculated not only by normal modes but also by higher order mode components that account for the foreshortening effect at beam-type structures. The approach is applied to a cantilever slender wing. Static and dynamic results are presented together with results from a commercial finite element solver and from the $U M / N A S T$ aeroelastic solver from the University of Michigan. The numerical study highlights the capability of the new approach to capture nonlinear effects while keeping the simplicity of the modal approach.
\end{abstract}

\section{Introduction}

THERE is a significant interest in the development of future passenger transport aircraft with lower emis1 sions and higher fuel efficiency. One of the key design features that supports achieving such goals is longer span wings, where induced drag is reduced. As the span increases along with the wing's aspect ratio, it leads to higher flexibility. This geometric effect becomes inevitable as light, high performance structural construction is used. The geometric nonlinear behavior on high-aspect-ratio wings can also be found in high-performance sailplanes and high-altitude long-endurance unmanned aerial vehicles. The increased structural flexibility is advantageous when dealing with external disturbances, such as gust encounters, due to the reduced rigid body acceleration. But it brings several challenges related to the modeling, analysis, and design for loads, aeroelastic stability, and flight dynamics and controls. It is critical that the geometrically nonlinear structural nature of the vehicle be taken into account in the earliest phases of design. ${ }^{1}$

Industry-standard aeroelastic simulation frameworks are typically based on a modal description of the airframe where eigenvectors and corresponding eigenvalues in the frequency range of interest are used to calculate structural deformations in a linear way. These have limited if any applicability for the problem of geometrically nonlinear aeroelasticity, where the emphasis turns to time-domain solutions and coupled aeroelasticity/flight dynamics analysis (due to the coupled nature of the rigid body and elastic response of the aircraft). Geometrically-exact beam-based formulations coupled with appropriate unsteady aerodynamics make the state of the art in solution frameworks for this type of problem. ${ }^{2}$ On the other hand, beam formulations may not be sufficient to model complex structural details of transport aircraft wings and the use of nonlinear FE codes for aeroelastic simulations can become very costly, especially for dynamic simulations.

\footnotetext{
*Research Engineer, AIAA Member (markus.ritter@dlr.de)

$\dagger$ Professor, Department of Aerospace Engineering, Fellow, AIAA (cesnik@umich.edu)

‡Professor, Department of Loads Analysis and Aeroelastic Design (wolf.krueger@dlr.de)
} 
The goal of this study is thus the development of a method that extends the classical modal approach towards large deformations while keeping its simplicity. Furthermore, the method should not be limited to beam type structures but should be applicable to any kind of FE models used for the dynamical modeling of aircraft. Its intended applications are steady and dynamic load and aeroelastic analysis.

Several authors have been working on methods for the reduced order modeling (ROM) of nonlinear structures. Mignolet and co-workers have given detailed descriptions for the derivation of static and dynamic nonlinear structural governing equations including quadratic and cubic stiffness coefficients. The determination of these stiffness coefficients (the ROM parameters) is done by a series of nonlinear static FE simulations with the full model using a commercial FE software. Their field of application ranges from plate structures to curved beams and the modeling of a complex UAV wing. ${ }^{3-5}$

Kuether and Allen describe methods based on nonlinear normal modes (NNMs) to simplify large, complex structures. NNMs basically describe the resonant frequency and response of a structure as a function of response amplitude or energy. The idea of their method is to assemble (nonlinear) structural subcomponents and determine their dynamical behaviour; the nonlinear dynamics of the assembly as a whole are predicted based on the dynamics of the subcomponents. ${ }^{6,7}$ Two substructuring methods that can be used in conjunction with detailed finite element models are presented by Kuether, ${ }^{6}$ where the second one is based on creating a reduced order model of a structure by applying a series of static loads to a nonlinear FE model. Quadratic and cubic stiffness terms are used to consider nonlinear force displacement behaviour and coupling of individual modes.

As will be shown, the kinematically nonlinear displacement field present in large deformations of wing-like structures can be reconstructed by shape functions of higher order. The method of quadratic components was successfully applied by Segalman and Dohrmann to improve the kinematical and dynamical description of rotating structures (beams) undergoing large displacements. ${ }^{8,9}$ This method is also used by van Zyl for the calculation of T-Tail flutter, where quadratic mode components enhance the description of kinematic relations at horizontal and vertical tail planes. ${ }^{10,11}$ In the approach presented, the concept of higherorder modes is further developed to better reconstruct nonlinear, large displacements. Higher-order stiffness

coefficients (derived here from a higher-order strain energy formulation) are also used to represent a nonlinear force-displacement behaviour.

\section{Derivation of the Method}

Compared to the classical modal approach, the proposed method is based upon the following extensions:

- Quadratic and cubic stiffness terms account for a nonlinear force-displacement relationship

- The reconstruction of the geometrically nonlinear displacement field is based on higher-order mode components

- The generalized forces are assumed to be a function of the state of deformation

- Inertia and stiffness terms in the dynamic formulation depend on the state of structural deformation and its rate

These extensions are discussed in details in the following subsections, where both the static and dynamic formulations are presented.

\section{II.A. Static formulation}

The derivation of the static governing equations involves two steps: First, a higher order formulation for the internal strain energy of the structure is given. Second, the geometrically nonlinear displacement field is reconstructed.

\section{II.A.1. Higher-order strain energy formulation}

The total potential energy of an elastic body $U$ consists of the sum of the total strain energy $U$ and the potential energy of the applied loads $V$. Furthermore, the total potential energy of the system is a constant. This statement is expressed as: ${ }^{12}$

$$
\pi=U+V=\text { const }
$$


Assuming linear elastic material behaviour (Hooke's law), and neglecting the influence of temperature, the total strain energy $U$ in the body is given as the following integral (here and what follows, the Einstein notation is used):

$$
U=\frac{1}{2} \int_{V} \tau_{i j} \epsilon_{i j} d v \quad(i, j=1,2,3),
$$

where $\tau_{i j}$ and $\epsilon_{i j}$ are the stress and strain component pairs, respectively. The work done by the applied loads $V$ can be expressed as:

$$
V=\int_{V} B_{i} u_{i} d v+\int_{S} T_{i}^{(\nu)} u_{i} d s
$$

where $B_{i}$ represents the components of the applied body forces, $u_{i}$ the components of the displacement field, and $T_{i}^{(\nu)}$ denotes the traction vector applied on the body surface. Tractions on the boundary can be related to stresses via Cauchy's formula: ${ }^{12}$

$$
T_{i}^{(\nu)}=\tau_{i j} \nu_{j}
$$

with $\nu$ as the unit outward normal vector.

Introducing a variation of both the total strain energy and the external work yields:

$$
\delta \pi=\delta(U+V)=\int_{V} \tau_{i j} \delta \epsilon_{i j} d v-\int_{V} B_{i} \delta u_{i} d v-\int_{S} T_{i}^{(\nu)} \delta u_{i} d s .
$$

Invoking the Principle of Minimum Total Potential Energy, one gets:

$$
\delta \pi=0
$$

Therefore, the variation of the total strain energy equals the negative variation of the external work:

$$
\delta U=-\delta V
$$

Introducing a discretization of the structure by finite elements with $N$ nodes, the work of the external forces is reduced to discrete nodal loads (forces and moments) denoted as $\mathbf{P}_{i}$ multiplied by generalized displacements (nodal translations and rotations) denoted as $\boldsymbol{\Delta}_{i}$. The total potential energy can then be given as follows:

$$
\pi=U-\sum_{k=1}^{N} \mathbf{P}_{k} \boldsymbol{\Delta}_{k}
$$

Taking the first variation of this expression leads to Castigliano's first theorem which states that the partial derivative of the strain energy $U$ with respect to an arbitrary displacement $\boldsymbol{\Delta}_{i}$ at a point $(i)$ equals the force $\mathbf{P}_{i}$ at this point in the direction of the displacement:

$$
\frac{\partial U}{\partial \boldsymbol{\Delta}_{i}}=\mathbf{P}_{i}(i=1, \ldots, N)
$$

The theorem similarly holds for nonlinear force-displacement relationships and can be generalized by the introduction of generalized displacements $\mathbf{q}$ and generalized forces $\mathbf{Q}:{ }^{12}$

$$
\frac{\partial U}{\partial \mathbf{q}_{i}}=\mathbf{Q}_{i}
$$

Now a vector $\psi$ that contains discrete displacements for every node of the structure is defined as a linear combination in the following way:

$$
\boldsymbol{\psi} \equiv \sum_{i=1}^{m} s_{i} \mathbf{a}^{i}
$$

where

$$
\mathbf{a}^{i}=\left(\boldsymbol{\Delta}_{1}, \ldots, \boldsymbol{\Delta}_{N}\right)^{T}
$$

contains constant (and for now arbitrary) displacements for each node and $s_{i}$ denotes a scalar value. The upper bound of the summation in Eq.(11), $m$, is equal to the number of generalized coordinates of the final 
governing equation that is to be derived in the following sections. Now the total strain energy, Eq.(2), is assumed to be a nonlinear and continuously differentiable function of the externally applied displacements $\psi$, which are a function of the scalar value $s_{i}$ only, and expanded in a Taylor series centered at zero (since the internal energy is zero for zero deformation):

$$
\begin{aligned}
U(\mathbf{s})= & \sum_{i=1}^{m} \frac{\partial U}{\partial s_{i}} s_{i} \\
& +\frac{1}{2 !} \sum_{i=1}^{m} \sum_{j=1}^{m} \frac{\partial^{2} U}{\partial s_{i} \partial s_{j}} s_{i} s_{j} \\
& +\frac{1}{3 !} \sum_{i=1}^{m} \sum_{j=1}^{m} \sum_{k=1}^{m} \frac{\partial^{3} U}{\partial s_{i} \partial s_{j} \partial s_{k}} s_{i} s_{j} s_{k} \\
& +\frac{1}{4 !} \sum_{i=1}^{m} \sum_{j=1}^{m} \sum_{k=1}^{m} \sum_{l=1}^{m} \frac{\partial^{4} U}{\partial s_{i} \partial s_{j} \partial s_{k} \partial s_{l}} s_{i} s_{j} s_{k} s_{l}+\text { h.o.t. }
\end{aligned}
$$

where: $\mathbf{s} \equiv\left(s_{1}, s_{2}, \ldots, s_{m}\right)^{T}$.

Differentiation of the strain energy defined in Eq.(13) yields the governing equation that comprises quadratic and cubic stiffness dependencies and accounts for a nonlinear force-deformation behaviour. The term $\frac{1}{2 !} \sum_{i=1}^{m} \sum_{j=1}^{m} \frac{\partial^{2} U}{\partial s_{i} \partial s_{j}}$ is comparable to the linear stiffness matrix. The Taylor series is carried out up to the fourth order so to capture up to cubic nonlinearities on the stiffness.

In general and supplementary to a deformation field as the one given by $\psi$, two approaches are possible in order to "excite" the structure considered and to identify the nonlinear force-displacement relations:

1. A deformation field can be imposed onto the structure degrees of freedom. A nonlinear static FE simulation is used to solve for the unknown forces at each grid point as a result of the applied deformations.

2. A force field can be imposed onto the structure. A nonlinear static FE simulation provides the corresponding deformation field.

In this work, the second approach was chosen due to the fact that the imposition of a forced field turned out to be more reliable than the imposition of a deformation field in a nonlinear structural solution. The question arises what "shape" the force field should have. In the preceding formulas, nodal coordinates were used. The goal of this work is to obtain a governing equation based on generalized coordinates with corresponding shape functions (eigenmodes in the linear case). In the following, the natural (in vacuo) eigenvalues and corresponding eigenvectors (mode shapes) of the structure are assumed to be available. Using one eigenvector, a physical force field (denoted as $\boldsymbol{F}^{i}$ ) can be defined in the following way:

$$
\mathbf{F}_{i}=\mathbf{K} \phi^{i} q_{i},
$$

where $\mathbf{K}$ denotes the nodal stiffness matrix of the structure, $q_{i}$ a scaling factor (for now), and $\phi^{i}$ denotes the column vector of one of the natural eigenvectors of the structure. Applying this force field in a nonlinear FE simulation, the resulting nodal displacements $u_{i}$ of the structure are a function of the scaling parameter $q_{i}$. Using a "small" value for $q_{i}$ (which yields small deformations), results in a deformation field that qualitatively equals the mode shape $\phi^{i}$ used. Increasing the value of $q_{i}$ in the nonlinear simulation at some point results in a deformation field that contains significant differences to the linear deformation field. This can be seen considerably if, e.g., the first bending mode shape is used as $\phi^{i}$. The linear part of the deformation field consists of pure transverse displacements, whereas for large values of $q_{i}$ in-plane motions become evident. For beam structures, this is known as the foreshortening effect. In summary, the following are the reasons for a force field (and especially one that is chosen according to Eq. 14) to "excite" the nonlinearities in a FE simulation:

1. Imposing a force field onto the structure and solving for the displacements in a nonlinear static FE simulation is easier than a imposing a displacement field and solving for the forces. 
2. The force field according to Eq.(14) yields a deformation field that equals the eigenvector $\phi^{i}$ for the linear case (small values of $q_{i}$ ).

3. In the nonlinear case (large $q_{i}$ ), nonlinear contributions in both the strain energy and the deformation field are excited.

For the nonlinear energy part, it is further considered in the following. If the structure is loaded by the force field defined in Eq.(14), the strain energy can be seen as a function of the scaling parameter $q_{i}$ (the nodal stiffness matrix and the eigenvector are constants). The Taylor series expansion of the internal strain energy as function of the scaling parameters $\boldsymbol{q}$ is then given by:

$$
\begin{aligned}
U(\boldsymbol{q}) & =\frac{1}{2 !} \sum_{i=1}^{m} \sum_{j=1}^{m} \frac{\partial^{2} U}{\partial q_{i} \partial q_{j}} q_{i} q_{j} \\
& +\frac{1}{3 !} \sum_{i=1}^{m} \sum_{j=1}^{m} \sum_{k=1}^{m} \frac{\partial^{3} U}{\partial q_{i} \partial q_{j} \partial q_{k}} q_{i} q_{j} q_{k} \\
& +\frac{1}{4 !} \sum_{i=1}^{m} \sum_{j=1}^{m} \sum_{k=1}^{m} \sum_{l=1}^{m} \frac{\partial^{4} U}{\partial q_{i} \partial q_{j} \partial q_{k} \partial q_{l}} q_{i} q_{j} q_{k} q_{l}+\text { h.o.t. }
\end{aligned}
$$

The term $\sum_{i=1}^{m} \frac{\partial U}{\partial q_{i}} q_{i}$ is omitted since no energy contribution as a linear function of $q_{i}$ exists. Using Castigliano's first theorem again and applying a differentiation with respect to $\boldsymbol{q}$ centered at zero yields the governing equation of the structure as a function of $\boldsymbol{q}$ :

$$
\begin{aligned}
\frac{\partial U}{\partial q_{p}} & =\sum_{i=1}^{m} \frac{\partial^{2} U}{\partial q_{i} \partial q_{p}} q_{i} \\
& +\frac{1}{2 !} \sum_{i=1}^{m} \sum_{j=1}^{m} \frac{\partial^{3} U}{\partial q_{i} \partial q_{j} \partial q_{p}} q_{i} q_{j} \\
& +\frac{1}{3 !} \sum_{i=1}^{m} \sum_{j=1}^{m} \sum_{k=1}^{m} \frac{\partial^{4} U}{\partial q_{i} \partial q_{j} \partial q_{k} \partial q_{p}} q_{i} q_{j} q_{k}+\text { h.o.t. }
\end{aligned}
$$

The following definitions are introduced:

$$
\begin{aligned}
{ }^{p} G_{1}^{i} & \left.\equiv \sum_{i=1}^{m} \frac{\partial^{2} U}{\partial q_{i} \partial q_{p}}\right|_{\boldsymbol{q}=0} \\
{ }^{p} G_{2}^{i j} & \left.\equiv \frac{1}{2 !} \sum_{i=1}^{m} \sum_{j=1}^{m} \frac{\partial^{3} U}{\partial q_{i} \partial q_{j} \partial q_{p}}\right|_{\boldsymbol{q}=0} \\
{ }^{p} G_{3}^{i j k} & \left.\equiv \frac{1}{3 !} \sum_{i=1}^{m} \sum_{j=1}^{m} \sum_{k=1}^{m} \frac{\partial^{4} U}{\partial q_{i} \partial q_{j} \partial q_{k} \partial q_{p}}\right|_{\boldsymbol{q}=0}
\end{aligned}
$$

The idea of the proposed method is to take the ${ }^{p} G_{n}$ stiffness matrices as generalized stiffnesses and the $\boldsymbol{q}$ as generalized coordinates. In this way, the following equation in the pseudo-generalized coordinates is obtained and used as the basis for the method:

$$
{ }^{p} G_{1}^{i} q_{i}+{ }^{p} G_{2}^{i j} q_{i} q_{j}+{ }^{p} G_{3}^{i j k} q_{i} q_{j} q_{k}=Q^{p} \quad(p=1, \ldots, m)
$$

Here the summation convention is used again. The stiffness parameters $G_{n}$ can be determined by polynomial fitting or numerical differentiation, as described below. In this example, a commercial finite element code was used for the modeling of the structure that provides the strain energy as part of the solution. A comparison at a typical beam model shows that the parameter $G_{1}^{i}$ matches the eigenvalue of the corresponding mode shape that is obtained from a real eigenvalue analysis. In this way, the linear case (structural governing equation in modal coordinates with linear terms only) is recovered by neglecting the terms of higher order in Eq.(21). The remaining issue is how the corresponding (linear and higher order) eigenvectors can be obtained. 


\section{II.A.2. Higher-order deformation reconstruction}

The second extension is the reconstruction of the geometrically nonlinear displacement field. As mentioned above, the nonlinear static FE analysis excites nonlinear terms in the strain energy, but also a nonlinear displacement field. This nonlinear displacement field is analyzed as described below.

For example, consider the mathematical model of a simple pendulum (as illustrated in Fig. 1). The kinematic relations in a Cartesian frame of reference are given by:

$$
v=\sin (\varphi) L ; \quad u=\cos (\varphi) L
$$

Assuming small values for the amplitude $\varphi$ of the pendulum, the small-angle approximation is used to

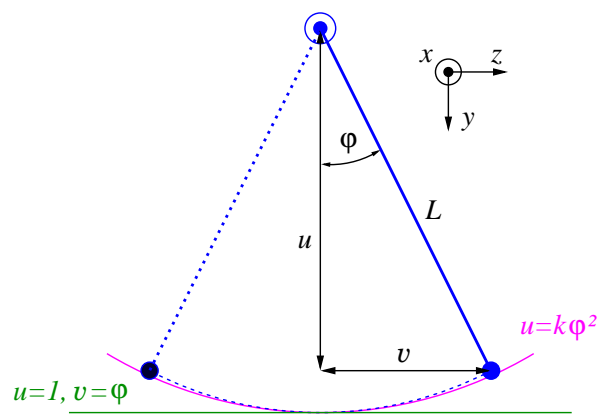

Figure 1. Kinematic relations on a simple pendulum, linear and parabolic approximation of the vertical tip displacement

simplify the analytical calculation of the pendulum's motion. This simplification makes use of the Taylor series expansion of the sine and cosine functions:

$$
\begin{aligned}
& \sin (\varphi)=\varphi-\frac{\varphi^{3}}{3 !}+\frac{\varphi^{5}}{5 !}+\text { h.o.t. } \\
& \cos (\varphi)=1-\frac{\varphi^{2}}{2 !}+\frac{\varphi^{4}}{4 !}+\text { h.o.t. }
\end{aligned}
$$

An extension of the small-angle approximation would consider the next terms of both the sine and cosine functions. The Method of the quadratic components applies this idea in structural dynamics, where the quadratic term of the Taylor series expansion of the cosine function is considered. This concept, which was introduced by Segalman and Dohrmann to analyze rotating flexible structures, provides a simple but efficient method to satisfy kinematic constraints up to second order. ${ }^{8,9,13}$ Another field of application in aeronautical engineering is the calculation of T-Tail flutter, where quadratic mode components are used to improve the description of kinematic relations at horizontal and vertical tail planes. ${ }^{10,11}$ A simplified illustration of the higher-order kinematic terms for the pendulum example is also shown in Fig. 1.

In the context of this work, the nodal deformation field as a result of a nonlinear mapping of the force field defined in Eq.(14) is expanded in a Taylor series centered at zero which is truncated after the fourth term:

$$
\begin{aligned}
\mathbf{u}(\boldsymbol{q})= & \sum_{i=1}^{m} \frac{\partial \mathbf{u}}{\partial q_{i}} q_{i} \\
& +\frac{1}{2 !} \sum_{i=1}^{m} \sum_{j=1}^{m} \frac{\partial^{2} \mathbf{u}}{\partial q_{i} \partial q_{j}} q_{i} q_{j} \\
& +\frac{1}{3 !} \sum_{i=1}^{m} \sum_{j=1}^{m} \sum_{k=1}^{m} \frac{\partial^{3} \mathbf{u}}{\partial q_{i} \partial q_{j} \partial q_{k}} q_{i} q_{j} q_{k} \\
& +\frac{1}{4 !} \sum_{i=1}^{m} \sum_{j=1}^{m} \sum_{k=1}^{m} \sum_{l=1}^{m} \frac{\partial^{4} \mathbf{u}}{\partial q_{i} \partial q_{j} \partial q_{k} \partial q_{l}} q_{i} q_{j} q_{k} q_{l}+\text { h.o.t. }
\end{aligned}
$$

The matrix $\mathbf{u}$ contains three columns for the displacements in $x, y$, and $z$ directions for each node of the structure. The constant term is omitted here. Partial differentiation of the displacement field defined in 
this way with respect to the generalized coordinates and substitution similar as defined above for the strain energy yields:

$$
{ }^{p} \boldsymbol{\Phi}=\frac{\partial \mathbf{u}}{\partial q_{p}}={ }^{p} \boldsymbol{\Phi}_{0}+{ }^{p} \boldsymbol{\Phi}_{1}^{i} q_{i}+{ }^{p} \boldsymbol{\Phi}_{2}^{i j} q_{i} q_{j}+{ }^{p} \boldsymbol{\Phi}_{3}^{i j k} q_{i} q_{j} q_{k}
$$

The term ${ }^{p} \boldsymbol{\Phi}_{0}$ can be seen as the equivalent of the structure's normal modes.

\section{II.A.3. Derivation of the generalized forces and final governing equation}

Considering Eq.(21), the forcing term of the structure's governing equation consists of the generalized force $Q^{p}$. In the linear case it is calculated as the product of the transposed of the eigenvalues and the discrete force field acting on the structure nodes. Applying the principle of virtual work and the Taylor series expansion of the higher order mode components results in an extension of the generalized forces. For simplicity, the approach described by van Zyl is followed and only the linear and the quadratic mode shape components are considered for the virtual work: ${ }^{11}$

$$
\delta V=\delta \mathbf{u}^{T} \boldsymbol{f}
$$

where $\boldsymbol{f}$ denotes an arbitrary nodal force field. Expansion of the virtual physical displacements $\delta \mathbf{u}$ using the linear and the quadratic mode shapes yields:

$$
\delta V=\delta q_{p}^{T}\left({ }^{p} \boldsymbol{\Phi}_{0}^{T}+{ }^{p} \boldsymbol{\Phi}_{1}^{i T} q_{i}\right) \boldsymbol{f}
$$

Thus the generalized forces are given as:

$$
Q^{p}={ }^{p} \boldsymbol{\Phi}_{0}^{T} \boldsymbol{f}+{ }^{p} \boldsymbol{\Phi}_{1}^{i^{T}} \boldsymbol{f} q_{i}
$$

Combining Eq.(21) and (32) yields:

$$
\left({ }^{p} G_{1}^{i}-{ }^{p} \boldsymbol{\Phi}_{1}^{i^{T}} \boldsymbol{f}\right) q_{i}+{ }^{p} G_{2}^{i j} q_{i} q_{j}+{ }^{p} G_{3}^{i j k} q_{i} q_{j} q_{k}={ }^{p} \mathbf{\Phi}_{0}{ }^{T} \boldsymbol{f}
$$

Eq.(33) is the static governing equation of the proposed method. Compared to the classical modal approach, it can be seen that the ${ }^{p} G_{1}^{i}$ term, which actually contains the eigenvalues of the linear, uncoupled system, is amended by the product of the transposed of the quadratic mode component matrix and the force field. This additional stiffness parameter is proportional to the force field applied to the structure and induces a coupling of the otherwise (in the linear sense) uncoupled eigenvectors. A nonzero value of the ${ }^{p} G_{2}^{i j}$ term indicates a non-isotropic structural behaviour. A force field applied to such a structure results in a softening or hardening in the corresponding direction of deformation, and a hardening or softening if the sign of the force field changes.

Following the solution of Eq.(33) by a nonlinear equation system solver, the nodal deformation field is reconstructed as function of the generalized coordinates $\boldsymbol{q}$ :

$$
\mathbf{u}(\boldsymbol{q})={ }^{p} \boldsymbol{\Phi}_{0} q_{p}+{ }^{p} \boldsymbol{\Phi}_{1}^{i} q_{p} q_{i}+{ }^{p} \boldsymbol{\Phi}_{2}^{i j} q_{p} q_{i} q_{j}+{ }^{p} \boldsymbol{\Phi}_{3}^{i j k} q_{p} q_{i} q_{j} q_{k},
$$

where the sum is taken again over repeated indices.

\section{II.B. Dynamic formulation}

The dynamic formulation is obtained by adding inertia terms to Eq.(33). Velocity-dependent forces (damping) are neglected here for simplicity. The governing equation is then given as:

$$
\boldsymbol{M} \ddot{q}_{i}+\left({ }^{p} G_{1}^{i}-{ }^{p} \boldsymbol{\Phi}_{1}^{i^{T}} \boldsymbol{f}\right) q_{i}+{ }^{p} G_{2}^{i j} q_{i} q_{j}+{ }^{p} G_{3}^{i j k} q_{i} q_{j} q_{k}={ }^{p} \mathbf{\Phi}_{0}{ }^{T} \boldsymbol{f},
$$

with $\boldsymbol{M}$ denoting the generalized mass matrix. In general, the mass matrix could be extended by considering higher order mode componets for the kinetic energy. This would yield a generalized mass which is function of the generalized coordinates and the generalized velocity of the system. This extension is considered as a topic of further research and not applied here. 


\section{II.C. Evaluation of the derivatives}

Both the nonlinear strain energy and the nonlinear deformation field are differentiated with respect to the scaling parameter $\boldsymbol{q}$ to calculate the stiffness terms ${ }^{p} G_{n}$ and the mode components ${ }^{p} \boldsymbol{\Phi}_{n}$. The extraction of these derivatives is involved. Several methods are applicable, essentially those used for numerical differentiation by finite differences. Formulas for the numerical evaluation of the components ${ }^{p} \boldsymbol{\Phi}_{0}^{i}$ and ${ }^{p} \boldsymbol{\Phi}_{1}^{i j}$ based on finite differencing are given in Dohrmann and Segalman. ${ }^{8,9}$ Expressions for the higher (third and fourth) order derivatives can be derived from Taylor series expansions.

The disadvantage of finite differences is the strong dependence on the stepping parameter that is used as well as the large effort which is involved in the implementation if expressions with higher order accuracy are desired. In this work, another approach was used for the evaluation of the energy and deformation field derivatives.

Applying the force field as defined in Eq.(14) from a nonlinear static FE simulation results in a corresponding value for the strain energy. Enhancing this equation and expressing it as polynomial function of two variables of degree four yields:

$$
\mathbf{F}^{i j}=\sum_{t=1}^{4}\left(s_{i}^{a} \phi^{i}+s_{j}^{b} \phi^{j}\right)^{t},(a, b=1, \ldots, \eta),(i, j=1, \ldots, m),
$$

where the parameters $s_{i}^{a}$ and $s_{j}^{b}$, which correspond to elements of the vector $\mathbf{s}$ defined in Eq.(13), can be seen as the scaling factors of two natural eigenvectors of the structure $\left(\phi^{i}\right.$ and $\left.\phi^{j}\right)$ that can be combined in this way. Therefore, a number of $\eta^{2}$ force fields is obtained for each combination of $i$ and $j$. These force fields are applied in a series of nonlinear static FE simulations and $\eta^{2}$ values for the strain energy or the deformation field are obtained. These solutions are collected in a vector $b_{i, j}$ :

$$
b^{i j}=\left[N_{1}\left(s_{i}^{1}, s_{j}^{1}\right) \ldots N_{\eta * \eta}\left(s_{i}^{\eta}, s_{j}^{\eta}\right)\right]^{T},
$$

where $N_{i}$ represents a nonlinear operator mapping the force field to a displacement field. A matrix $A$ can be defined that contains the values that were chosen for the parameters $s_{i}^{a}$ and $s_{j}^{a}$ in its a-th row, i.e.,

$$
A_{a}=\sum_{t=1}^{4}\left(s_{i}^{a}+s_{j}^{a}\right)^{t}
$$

This matrix contains 14 columns since the polynomial defined in Eq.(36) contains 14 terms. Using the matrix $A$ and the vector $b$, a linear system with $\eta^{2}$ equations can be defined:

$$
A x=b
$$

where the solution vector $x$ contains 14 unknown coefficients which are to be determined.

The system of equations in Eq.(39) may be overdetermined, depending on the number of scaling parameters $\eta$ that are used for the nonlinear FE simulations. A solution is found by calculating the pseudo-inverse of matrix A. This corresponds to an optimization problem, where the polynomial defined in Eq.(36) that depicts a surface in two dimensions is fitted into the solutions of the vector $b$ by minimizing an error norm. Higher precision can easily be obtained by the use of more points, meaning a higher value for $\eta$. Once the vector of coefficients, $x$, is determined, the derivatives are easily obtained by differentiation of the polynomial defined in Eq.(36). Coupled derivatives are evaluated in a similar way. The dependence of the derivatives of the strain energy and the deformation field on the value of $\eta$ is shown in the sections below for a representative beam type structure. It must be mentioned that by using a polynomial which is a function of two variables, only two modes can be coupled. The cubic stiffness matrix and the fourth order mode component defined in Eq.(33) contains mixed partial derivatives with respect to three variables and thus a coupling of three modes can be considered. For simplicity, only two modes are taken into account in the formulation presented here. An upgrade to three coupled modes by an expansion of the polynomial in Eq.(36) is straightforward, but the cost for the evaluation of all derivatives increases. Both the strain energy and the deformation field that are obtained from one FE simulation can be differentiated simultaneously with respect to the scaling parameters $s_{i}$ and $s_{j}$. Due to Schwartz's exchange theorem the matrices containing the mixed derivatives are symmetric. ${ }^{8}$ 


\section{Numerical Studies}

The results of selected static and dynamic, as well as of static aeroelastic coupled simulations obtained with the described approach are presented in the following sections. Furthermore, the test case presented in the following sections was modelled with University of Michigan's UM/NAST solver. This toolbox provides excellent reference data in terms of nonlinear structural dynamics and coupled aeroelastic simulations. ${ }^{14}$

The test case consists of a highly flexible beam model with a length of 16 meters to resemble a slender generic aircraft wing. It is discretized by 32 beam finite elements and 33 nodes. The moments of inertia vary quadratically along the beam axis. This setup was used to obtain bending deformations with constant curvature for typical aerodynamic loadings. Discrete mass elements with prescribed mass moments of inertia were used at each structural node to tune the dynamic structural behaviour. The layout of the model is shown in Fig. 2. Displacements of node 33 (outermost node) will be used to show the behaviour of the beam and the method for dynamic loadings. For all simulations with the proposed method, only seven selected mode

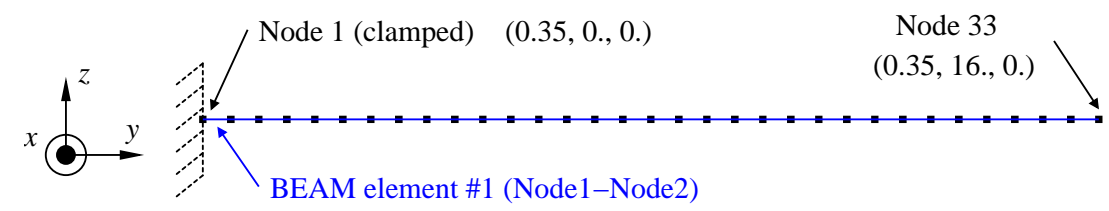

Figure 2. $16 \mathrm{~m}$ beam FE model, 33 Nodes, clamping at Node 1

shapes were used. Thus the higher order stiffness matrices and mode components ( $G$ and $\boldsymbol{\Phi}$, respectively) were calculated for these modes. The selected modes were found to be sufficient to obtain convergence in terms of the static deformation field; also the computational effort is reduced when considering less modes especially for the dynamic simulations. The values of ${ }^{p} G_{1}^{i}$ and ${ }^{p} \boldsymbol{\Phi}_{1}^{i}$ reconstructed with the method described in subsection II.C are very close to the corresponding values from the natural eigenvalues of the beam. Table 1 lists the types and frequencies of the ten lowest mode shapes of the beam model obtained from the finite element modal analysis. Additionally, the eigenvalues from the reconstruction method are given for the seven selected modes. The second, third, and fourth in-plane bending mode were omitted, since their contribution to a deformation from a typical aerodynamic loading was expected to be negligible. For

Table 1. Eigenvalues of the ten lowest modes of the beam model; values were obtained from a FE analysis and from a polynomial reconstruction method.

\begin{tabular}{l|l|c|c} 
Mode & Type of mode & Eigenvalue FE analysis $[\mathrm{Hz}]$ & Eigenvalue reconstructed $[\mathrm{Hz}]$ \\
\hline 1 & first bending & 0.595 & 0.595 \\
2 & first bending in-plane & 1.190 & 1.190 \\
3 & second bending & 2.705 & 2.705 \\
4 & second bending in-plane & 5.407 & - \\
5 & third bending & 6.956 & 6.956 \\
6 & fourth bending & 13.358 & 13.358 \\
7 & third bending in-plane & 13.893 & - \\
8 & fifth bending & 21.908 & 21.908 \\
9 & fourth bending in-plane & 26.651 & - \\
10 & first torsion & 27.132 & 27.132
\end{tabular}

isotropic structures (as is the case for this beam model) the ${ }^{p} G_{2}^{i j}$ term becomes zero. The diagonal values of the cubic stiffness ${ }^{p} G_{3}^{i j k}$ have very small values for this model (on the order of $10^{-3}$ for the first bending mode). 


\section{III.A. Static simulations and validation with nonlinear data from UM/NAST}

A constant tip force in the $z$ direction was applied at the outermost node of the beam with assumed values between $500 \mathrm{~N}$ and $3000 \mathrm{~N}$ to simulate a constant static loading. The static governing equation is nonlinear and was solved using a root-finding algorithm with a specified start estimate. The results of this test case are presented in Fig. 3. It must be mentioned that no follower forces and no gravity have been considered in the static solutions. In a fully nonlinear solution sequence (considering incremental loads), the force applied to the beam is increased stepwise and equilibrium is ensured in every step until the specified load is applied. In the solution sequence used for the proposed method, the specified value of the force is applied always onto the undeformed initial configuration of the beam. The inclusion of follower forces in the solution sequence is subject to further research. The values were chosen to excite deformations of the beam beyond the limit of

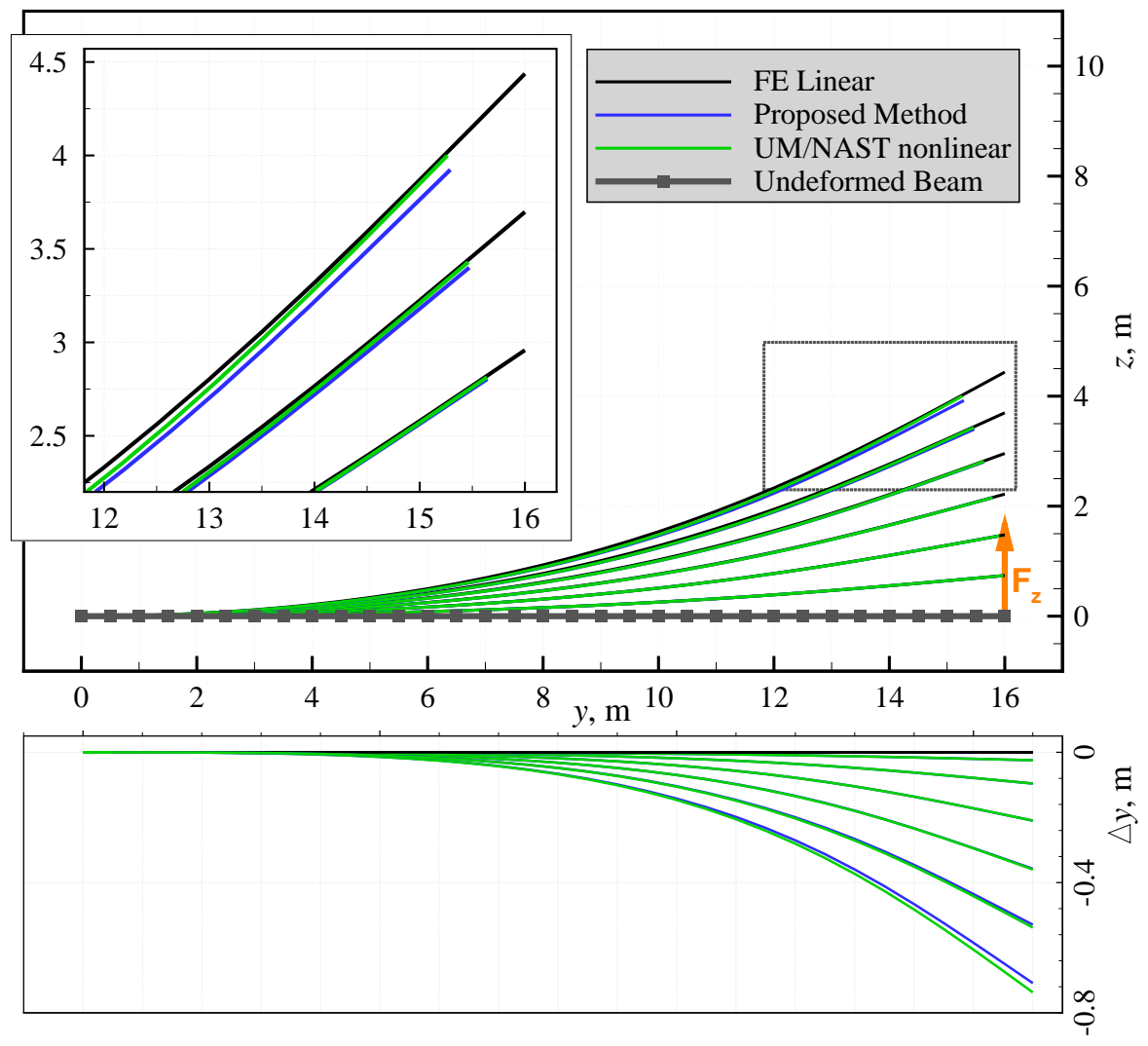

Figure 3. Comparison of the static displacement fields obtained by different methods for forces with values of 500, $1000,1500,2000,2500$, and $3000 \mathrm{~N}$ applied along the $z$ direction at the end of the beam.

structural linearity. This can be seen in terms of the displacement of the beam in $z$ and in $y$ directions. The linear FE solution yields too large displacements in the $z$ direction and of course omits the displacement in the $y$ direction (foreshortening effect) completely. The results of the proposed method are in good agreement with the nonlinear reference data from UM/NAST, and the displacement in the $z$ and in the $y$ directions are captured well up to the tip force of $2500 \mathrm{~N}$. Differences between the enhanced modal approach and the nonlinear reference data become larger starting at the tip force of $3000 \mathrm{~N}$. For this force, the bending deformation in the $z$ direction reaches a value of $25 \%$ of the span of the beam. An interesting point is that the nonlinear deformation fields are "on top" of the linear one but with the foreshortening effect keeping the beam length constant.

\section{III.B. Dynamic simulations and validation with nonlinear data from UM/NAST}

The dynamic validations of the method omitting displacement dependent mass and velocity dependent stiffness terms were done in two steps. First, a constant force (as for the static simulations) was applied onto the 

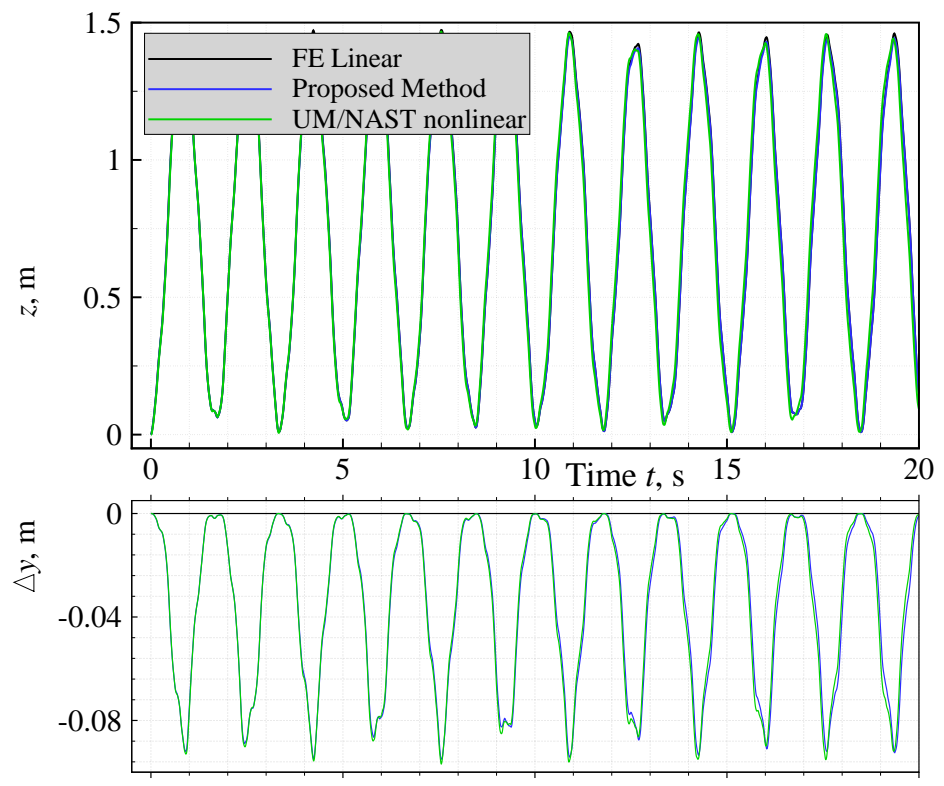

Figure 4. Time function of the displacement of the beam's tip node for a step force of $500 \mathrm{~N}$ at the wingtip along the $z$ direction.

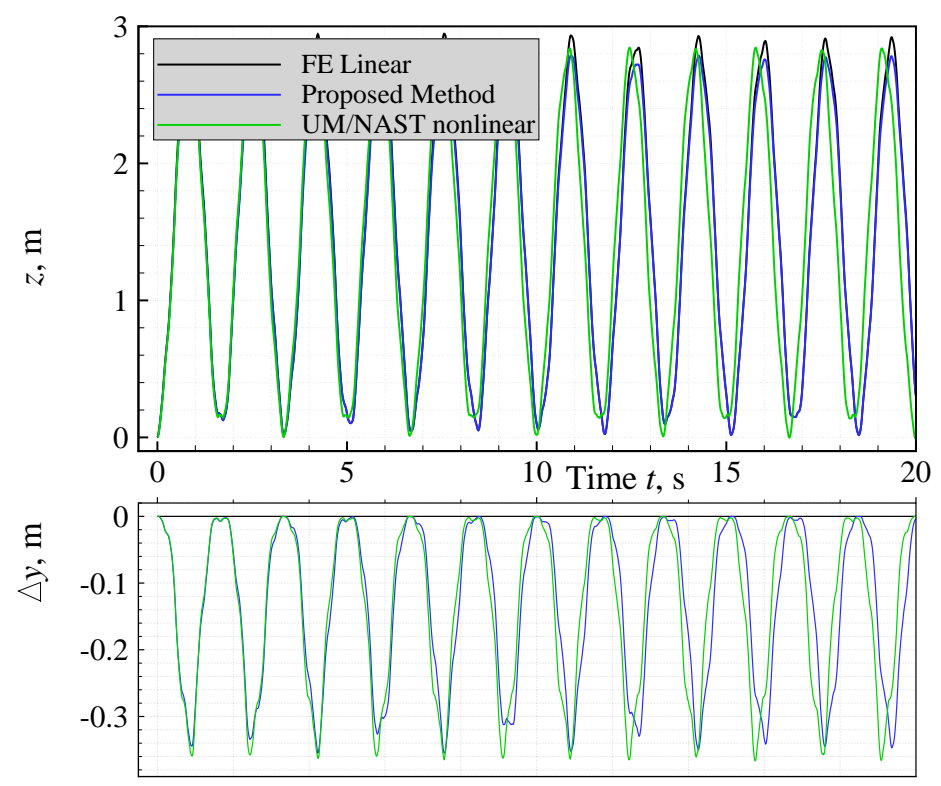

Figure 5. Time function of the displacement of the beam's tip node for a step force of $1000 \mathrm{~N}$ at the wingtip along the $z$ direction.

outermost node of the beam and the dynamic governing equation was solved numerically. This was done using an implicit multistep backward differentiation (BDF) scheme with variable time step size. No structural damping, no follower forces, and no gravity were considered. The force was chosen to excite deformations with pronounced nonlinearities. Step function loads of $500 \mathrm{~N}$ and $1000 \mathrm{~N}$ along the $z$ direction were applied at the outermost node of the beam. The tip response from these simulations are shown in Figs. 4 and 5 for the respective force. As for the static simulations, the displacements of the proposed method are in good agreement with the nonlinear reference data from UM/NAST, both in the $z$ and the $y$ directions. However, the linear and the enhanced modal approach yield a slightly lower frequency than the results from the UM/NAST solver. This can be explained by the fact that in the nonlinear solution sequence of UM/NAST, forces are applied at the current (deformed) configuration. As the bending deformation of the beam reaches larger values, the force on the tip can be split up into a component acting perpendicular to the beam and 
a component acting in the longitudinal axis of the beam. The second component slightly stiffens the beam and thus increases the frequency of the beam's oscillation. For the proposed method, the forces are always applied at the undeformed configuration and thus this stiffening effect is not captured.

Next, a sinusoidal tip force of $1000 \mathrm{~N}$ with a frequency of $0.8 \mathrm{~Hz}$ was applied at the outermost node of the beam model to evaluate its dynamic response. As before, an implicit BDF scheme was used to solve the dynamic governing equation. No structural damping, no follower forces, and no gravity were considered in all solvers. The initial conditions for the generalized displacements and the generalized velocities were set to zero. The results are given in Fig. 6 in terms of the displacement in the $z$ and in the $y$ directions of the outermost node. It can be seen that all solutions yield comparable results for the displacements in the $z$
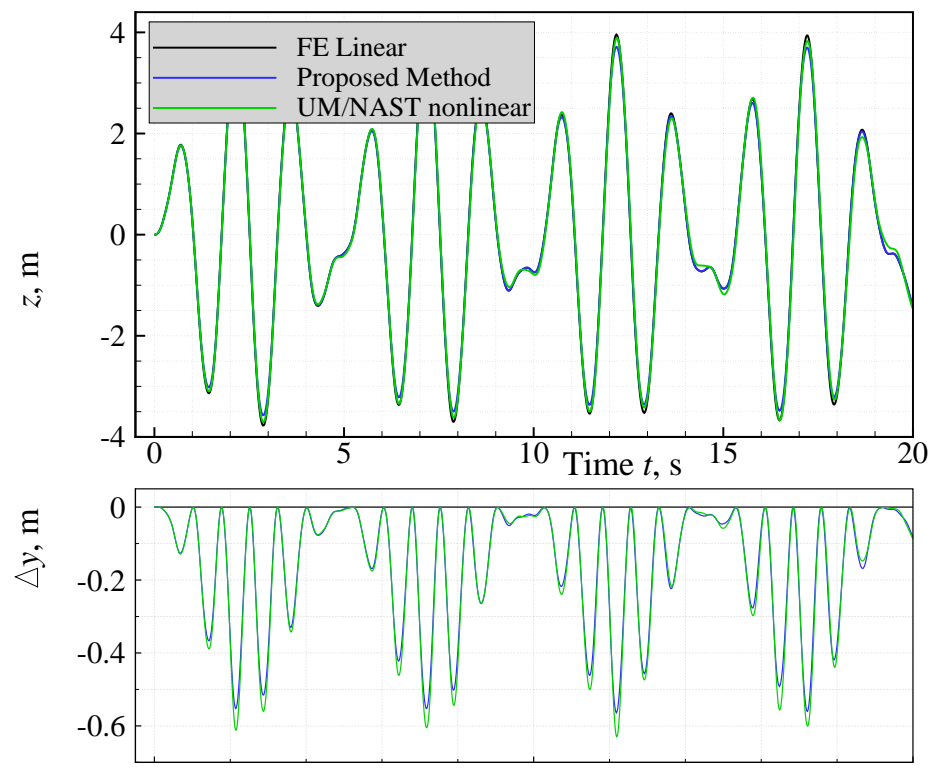

Figure 6. Comparison of the dynamic displacement field obtained by different methods for a sinusoidal tip force applied at the end of the beam. Force amplitude $=1000 \mathrm{~N}$, frequency $=0.8 \mathrm{~Hz}$.

directions. Slight differences occur at the largest values (about 4 meters) for the linear FE results, which gives too high deformations (comparable to the static results). As for the static case, the deformation in the $y$ direction is reflected by the proposed method even though smaller differences occur. The effect of frequency reduction in the results of the proposed method is less pronounced here due to the fact that the force is not constant anymore but varies with time and thus the time-dependent maximum of the force not always coincides with the maximum deformation of the outermost node. To further evaluate the dynamic behaviour of the method at slightly higher frequencies, two more dynamic simulations are presented with tip forces of $2000 \mathrm{~N}$ and frequencies of $1.0 \mathrm{~Hz}$ and $2.0 \mathrm{~Hz}$, respectively. The results are shown in Figs. 7 and 8. Despite a good overall agreement between the UM/NAST results and the results of the proposed method, differences occur both for the deformation in the $z$ and in the $y$ direction for the $1.0 \mathrm{~Hz}$ test case. The $2.0 \mathrm{~Hz}$ test case shows maximum tip deflections in $z$ direction of only 1.2 meters (which is in the linear regime) and exposes larger differences between the methods. It must be mentioned that the results of the proposed method were calculated including only five bending modes and that for this test case the deformations include higher frequency components, as can be seen in Fig. 8. Further simulations including more bending modes with higher frequencies could yield better results for the proposed method with this test case.

\section{III.C. Static aeroelastic simulations and validation with UM/NAST results}

For the enhanced modal approach, a vortex-lattice solver was used that provides the aerodynamic forces. The solver is implemented such that the aerodynamic panels can undergo any kind of translation and rotation due to elastic deformations. The linear potential equations are solved for the circulation at each panel given a prescribed downwash at each panel's collocation point. The force produced by each panel is calculated 

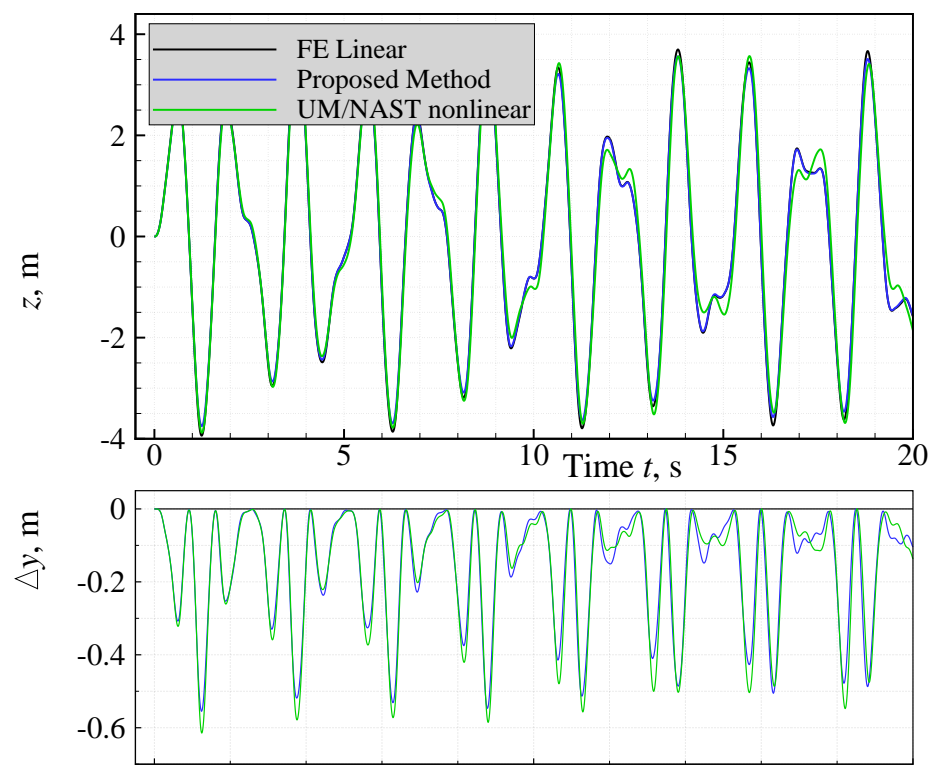

Figure 7. Comparison of the unsteady displacement field obtained by different methods for a sinusoidal tip force applied at the end of the beam. Force amplitude $=2000 \mathrm{~N}$, frequency $=1.0 \mathrm{~Hz}$.
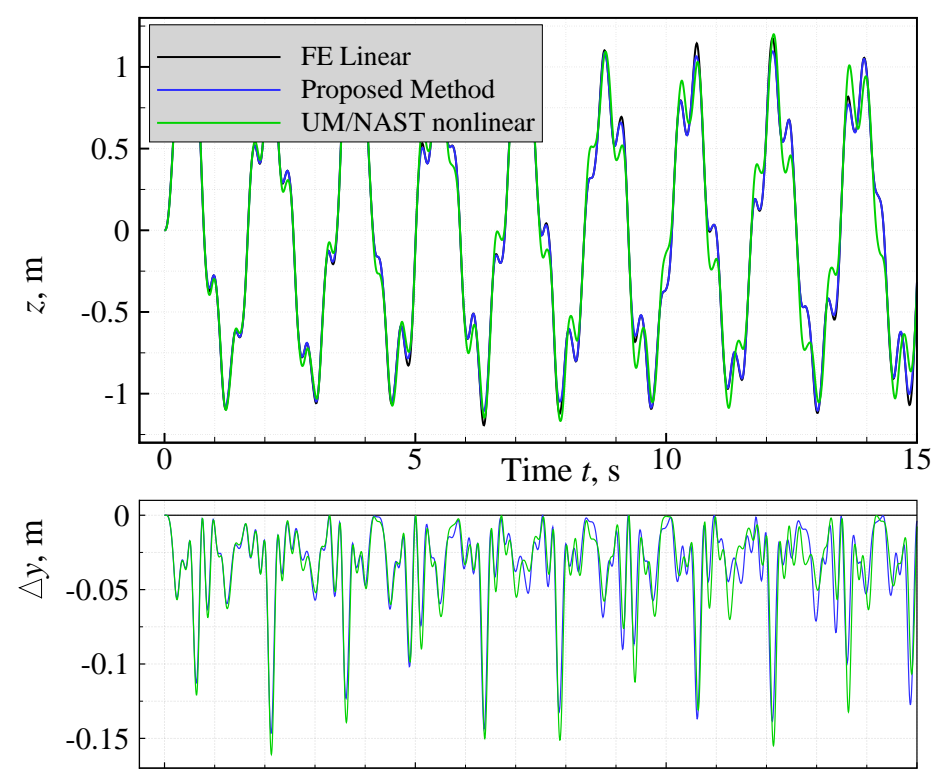

Figure 8. Comparison of the unsteady displacement field obtained by different methods for a sinusoidal tip force applied at the end of the beam. Force amplitude $=2000 \mathrm{~N}$, frequency $=2.0 \mathrm{~Hz}$.

based on the current geometry of the panel, the circulation and the onflow conditions (velocity and fluid density).

The force transfer from the aerodynamic panels onto the structural grid is done via the transposed of the coupling matrix. The coupling matrix itself is build from radial basis functions, a similar approach is used in commercial aeroelastic codes. ${ }^{15}$ Transforming aerodynamic forces into equivalent forces on the beam's nodes requires the calculation of forces and moments. In this case, another approach was used to avoid the calculation of moments. A so-called coupling model was built which uses rigid-bar elements that are connected to a beam node at one side each. The other end of the rigid-bar elements are used as coupling points to where the aerodynamic forces are transferred. The rigid-bar elements were included in the modal analysis of the beam and in the reconstruction of the higher-order mode components. Thus the beam model 
was enhanced into a "volume model" with the result that no moments have to be calculated and the common coupling matrix approach can be used for the force transfer.

Still using the 16-meters-span, one meter chord wing example, the aerodynamic grid is discretized by 64 panels in the spanwise and 16 panels in the chordwise directions. A layout of the aerodynamic grid together with the beam's reference axis is shown in Fig. 9. The coupling approach described using a coupling model based on rigid-bar elements is depicted in Fig. 10. The onflow velocity was fixed to $40 \mathrm{~m} / \mathrm{s}$, the reference

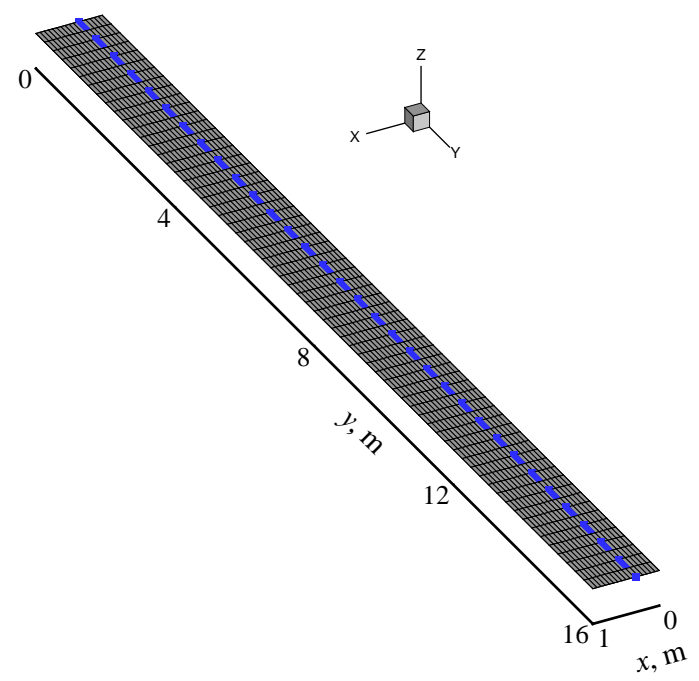

Figure 9. VL grid discretized by panels with the beam model's reference axis used for the static coupling simulations.

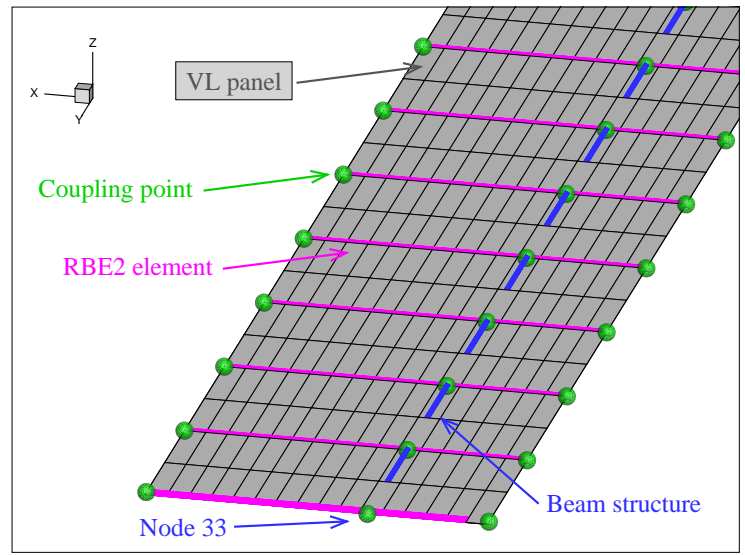

Figure 10. Depiction of the coupling approach: Aerodynamic forces are transformed onto the structure's coupling points and then transferred implicitly to the beam's nodes via rigid-bar elements.

density of the fluid (air) is $1.225 \mathrm{~kg} / \mathrm{m}^{3}$. To obtain deformations of the coupled aeroelastic system ranging from the linear to the nonlinear regime, the root angle of attack (AoA) was varied within the range of one to five degrees in steps of one degree. UM/NAST uses a strip-theory aerodynamic model. However, UM/NAST enables the correction of the static force produced by each strip by considering a weighting factor that accounts for wingtip effects. The correction factors for UM/NAST were taken from the lift distribution given by the vortex-lattice solver for the rigid wing. This correction method results in identical aerodynamic forces for the vortex-lattice and the UM/NAST aerodynamic solvers for the undeformed wing.

Results of the static coupling simulations are shown in Fig. 11 in terms of the bending deformations of the beam. For this test case, results from Nastran SOL144 solution sequence (static aeroelastic) are also presented. The level of difficulty is increased for these simulations compared to the static simulations with a constant force in the $z$ direction, since the forces on the nodes now have contributions along the $y$ direction as well. The bending deformation from the proposed method agrees well with the results form UM/NAST. At higher angles of attack, the difference between the linear and the nonlinear solutions becomes larger and the 
beam tends to have a reduced deformation in the UM/NAST results. This can be seen as realistic, since the forces are always applied onto the undeformed structure (in the linear and the enhanced modal approach), but the enhanced modal approach considers the "in-plane" force due to the quadratic mode component.

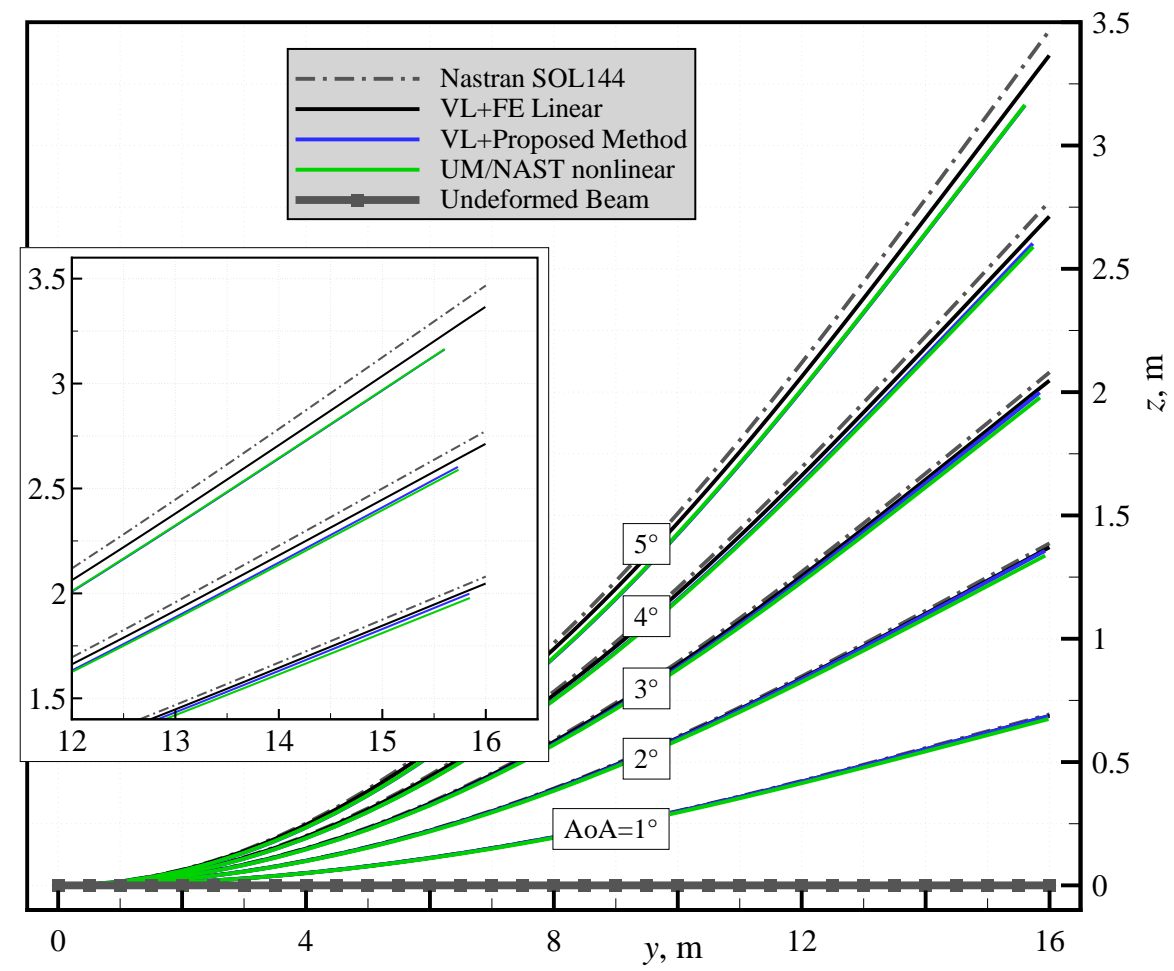

Figure 11. Comparison of static coupling results calculated by the proposed method coupled with a vortex-lattice solver and by UM/NAST.

\section{Concluding Remarks}

A method was presented that enhances the classical modal approach used in structural dynamics and aeroelastic applications towards large geometric deformations. The goal was to obtain a method that can be applied to any kind of FE model (such as the modal approach) and considers nonlinearities in the forcedisplacement relationships and in the deformation field. It must be mentioned that this method is supposed to be used for (slender) wing-like structures rather than for short plates. The derivation of the method is based on the assumption that both the strain energy and the displacement field of the structure due to typical loadings contain pronounced higher order terms. These higher order terms have to be evaluated for the system considered. Based on these terms, nonlinear static and dynamic governing equations were obtained in which the generalized stiffnesses are depending on the forces applied to the structure. In principle, additional higher-order mode components could be used for an even better reconstruction of the nodal displacement field. The proposed method was applied to a beam-type structural model resembling a generic slender wing. The static and dynamic results showed that the proposed method can acceptably reflect the geometrically nonlinear deformation field which occurs at large bending deformations up to values of approximately $25 \%$ of the beam's length. Static aeroelastic simulations show that the method surpasses the linear modal approach by considering lateral forces in the solution which arise from inclined panels due to the deformation of the wing.

However, different issues with the method require further studies. Mainly the question regarding how a displacement and velocity dependent generalized mass matrix can improve the dynamic results. The inclusion of follower-type forces and an incremental load solution approach will be done as next steps. Further aeroelastic simulations with the method will be done with the focus on dynamic excitations due to gust encounter. Also more complex models such as wingboxes discretized by plate-type FE elements will be simulated with this approach. 


\section{Acknowledgments}

A part of this work was carried out during an internship of the first author at the Active Aeroelasticity and Structures Research Lab at the University of Michigan. The authors would like to thank the entire team of the laboratory for their support and especially Ryan Kitson for providing the UM/NAST results. Furthermore, the support of the DLR Institute of Aeroelasticity for this work is gratefully acknowledged.

\section{References}

${ }^{1}$ Cesnik, C. E. S., Palacios, R., and Reichenbach, E. Y., "Reexamined Structural Design Procedures for Very Flexible Aircraft," Journal of Aircraft, Vol. 51, No. 5, 2014, pp. 1580-1591.

${ }^{2} \mathrm{Su}$, W. and Cesnik, C. E. S., "Strain-Based Analysis for Geometrically Nonlinear Beams: A Modal Approach," $53^{\text {rd }}$ AIAA/ASME/ASCE/AHS/ASC Structures, Structural Dynamics and Materials Conference, Honolulu, Hawaii, April 2013.

${ }^{3}$ Mignolet, M. P., Przekop, A., Rizzi, S. A., and Spottswood, S. M., "A review of indirect/non-intrusive reduced order modeling of nonlinear geometric structures," Journal of Sound and Vibration, Vol. 332, No. 10, 2013, pp. 2437 - 2460.

${ }^{4}$ Kim, K., Radu, A. G., Wang, X. Q., and Mignolet, M. P., "Nonlinear reduced order modeling of isotropic and functionally graded plates," International Journal of Non-Linear Mechanics, Vol. 49, No. 0, 2013, pp. 100 - 110.

${ }^{5}$ Wang, X. Q., Perez, R. A., and Mignolet, M. P., "Nonlinear Reduced Order Modeling of Complex Wing Models," 54 ${ }^{t h}$ AIAA/ASME/ASCE/AHS/ASC Structures, Structural Dynamics, and Materials Conference, Boston, Massachusetts, Apr 2013.

${ }^{6}$ Kuether, R. J. and Allen, M. S., "Nonlinear Modal Substructuring of Systems with Geometric Nonlinearities," Structures, Structural Dynamics, and Materials and Co-located Conferences, 54 ${ }^{\text {th }}$ AIAA/ASME/ASCE/AHS/ASC Structures, Structural Dynamics, and Materials Conference, Boston, Massachusetts, Apr 2013.

${ }^{7}$ Kuether, R. J. and Allen, M. S., "Substructuring with Nonlinear Reduced Order Models and Interface Reduction with Characteristic Constraint Modes," AIAA SciTech, $55^{\text {th }}$ AIAA/ASME/ASCE/AHS/ASC Structures, Structural Dynamics, and Materials Conference, National Harbor, Maryland, Jan 2014.

${ }^{8}$ Segalman, D. J. and Dohrmann, C. R., "Dynamics of Rotating flexible Structures by a Method of Quadratic Modes," Sandia National Laboratories, Structural Dynamics Division, Albuquerque, New Mexico, 1990.

${ }^{9}$ Segalman, D. J. and Dohrmann, C. R., "A Method for Calculating the Dynamics of Rotating Flexible Structures, Part1: Derivation," ASME Journal of Vibration and Acoustics, Vol. 118, 1996, pp. 313-317.

${ }^{10}$ van Zyl, L. H., Sutherland, A. N., and Rossouw, P. S., "Parabolic Mode Shapes: What they are, where to get them and what to do with them," International Forum on Aeroelasticity and Structural Dynamics, Seattle, Washington, 2009.

${ }^{11}$ van Zyl, L. H. and Mathews, E. H., "Quadratic Mode Shape Components From Linear Finite Element Analysis," ASME Journal of Vibration and Acoustics, Vol. 134, Feb. 2012.

${ }^{12}$ Shames, I. H. and Dym, C. L., Energy and Finite Element Methods in Structural Mechanics, Hemisphere Publishing Corporation, 1985.

${ }^{13}$ Robinett, R. D., Feddema, J., Eisler, G. R., Dohrmann, C., Parker, G. G., Wilson, D. G., and Stokes, D., "The Method of Quadratic Modes," Flexible Robot Dynamics and Controls, Vol. 19, IFSR International Series on Systems Science and Engineering, 2002.

${ }^{14} \mathrm{Su}$, W. and Cesnik, C. E. S., "Nonlinear Aeroelasticity of a Very Flexible Blended-Wing-Body Aircraft," Journal of Aircraft, Vol. 47, No. 5, Sep 2010, pp. 1539-1553.

${ }^{15}$ Anon., MSC Nastran 2012 Quick Reference Guide, MacNeal-Schwendler Corporation, 2011. 